\title{
Cyclic guanosine monophosphate inhibits the palmitoylation of LETM1 and downregulates the activity of AKT in human pulmonary artery smooth muscle cells
}

\section{Wenqing Li}

Beijing Hospital

Wei Huang

Beijing Hospital

Ran Miao

Beijing Chao-Yang Hospital: Beijing Chaoyang Hospital

Lihui Zou

Beijing Hospital

Fei Xiao ( $\nabla$ xiaofei3965@bjhmoh.cn )

Beijing Hospital https://orcid.org/0000-0003-2490-850X

\section{Research Article}

Keywords: cGMP, LETM1, palmitoylation, AKT, HPASMCs

Posted Date: January 25th, 2022

DOI: https://doi.org/10.21203/rs.3.rs-1252716/v1

License: (c) (1) This work is licensed under a Creative Commons Attribution 4.0 International License. Read Full License 


\section{Abstract}

Cyclic guanosine monophosphate (cGMP) is a drug target for the treatment of pulmonary arterial hypertension (PAH). However, the molecular mechanism of cGMP in the pathogenesis of PAH remains unclear, and long-term use of cGMP target drugs leads to drug resistance. We tried to explore the new targets downstream of cGMP signaling and develop novel therapies for PAH. We combined mass spectrometry (MS)-based quantitative proteomics with click-chemistry-probes to identified palmitoylated candidate proteins. S-palmitoylation assay was ultilized to test whether the palmitoylation level of the candidates were regulated by cGMP. We identified palmitoylated proteins in human pulmonary artery smooth muscle cells (HPASMCs). Functional enrichment of the palmitoylated proteins showed that 52 proteins were involved in transmembrane transport, of which 7 were engaged in mitochondrial transport. S-palmitoylation assay in vitro demonstrated that the inner mitochondrial membrane protein, LETM1, was palmitoylated in the cytoplasmic domain at C-terminus. More importantly, cGMP could inhibit the palmitoylation level of LETM1, which in turn suppressed the activity of AKT regulated by LETM1. Our study found that the palmitoylation of LETM1 was the new target downstream of cGMP signaling. The palmitoylated site of LETM1 may provide a new direction for the development of novel therapies for PAH. Moreover, the palmitoylation level of LEMT1 may be a new biomarker for the analysis of prognosis.

\section{Introduction}

Cyclic guanosine monophosphate (cGMP) is the second messenger of nucleotides, which was first identified about a half a century ago [1]. cGMP originates from guanylyl cyclases (GC) catalyze by natriuretic peptide or nitric oxide (NO) and transduces its effects by downstream phosphodiesterases (PDE) and protein kinases [2]. cGMP regulates many cellular functions, ranging from contractility to growth. The NO/cGMP signaling pathway is essential for the maintenance of vascular tone and dysfunction of the cGMP signaling leads to a number of cardiovascular diseases, such as pulmonary arterial hypertension (PAH) [3]. PAH is a progressive disease characterized by elevated pulmonary arterial pressure, resulting in pulmonary vasoconstriction, thrombosis in situ, and even right heart failure and death [4]. cGMP is the main target of drug therapy for PAH, but long-term use of cGMP targeted drugs is prone to drug resistance. At the same time, these drugs promote peripheral vasodilation, which will lead to the side effects of systemic hypotension. In addition, the molecular mechanism of cGMP in the pathogenesis of PAH remains elusive.

Protein palmitoylation is a post-translational modification of proteins with fatty acids, which plays significant part in eukaryotic physiology and human pathology by regulating protein localization, trafficking, stability, assembly, and protein-protein interaction [5, 6]. Protein palmitoylation involves the covalent connection of a 16-carbon fatty acid moiety, palmitate, and amino acid residues, such as cysteines (S-palmitoylation), serines/threonines (0-palmitoylation) and lysines (N-palmitoylation) [7]. Among them, only S-palmitoylation is reversible, which is catalyzed by protein acyl-transferases with AspHis-His-Cys (DHHC) motifs and removed by acyl-protein thioesterases [8]. Based on the strict regulation for activity, researchers pay more attention to S-palmitoylation in the last 30 years and detect more than 
400 palmitoylated proteins, including both soluble proteins and integral membrane proteins [9]. For soluble proteins, such as $\mathrm{H}$-Ras and $\mathrm{N}$-Ras, palmitoylation facilitates the localization to lipid rafts and plasma membranes by enhancing the hydrophobicity of them [10]. For integral membrane proteins, palmitoylation occurs not only in cytoplasmic tails or loops but also in the vicinity or within transmembrane domains [11]. Due to the difficulties in analyzing a lipid modification and dealing with transmembrane domains, more studies are now focused on the cytoplasmic, including receptors, channels and transporters. Numerous channels or transporters are essential for controlling the physiology and pathology of tissues through the transmembrane transport of ions, proteins, metabolic products, etc., and the activities of some of them are regulated by palmitoylation. For example, $\mathrm{P} 2 \mathrm{X}_{7}$ is a non-selective cation channel activated by extracelluar ATP. Cryo-electron microscopy structures of rat $\mathrm{P} 2 \mathrm{X}_{7}$ show that palmitoylation is observed in the cytoplasmic element, c-cysteine anchor, which prevents desensitization by anchoring the pore-lining helix to the membrane [12]. Another case is about the transmembrane sodium-calcium exchanger 1 (NCX1), which facilitates the bidirectional transport of calcium levels by the gradient of transmembrane sodium. The palmitoylation of NCX1 is localized in the intracellular loop and the inactivation of NCX1 is controlled by modifying the NCX1 dimer's structure [13]. Based on the regulatory mechanism of palmitoylation on transmembrane transport, the pharmacological targets of palmitoylation sites can be used as a way of clinical treatment of related diseases. Considering the key role of palmitoylation and the limitations of cGMP targeted therapy for PAH, the present study aimed to identify the cGMP-regulated palmitoylated proteins.

In this study we combined mass spectrometry (MS)-based quantitative proteomics with click-chemistryprobes to quickly identified palmitoylated candidate proteins that maybe regulated by cGMP in cell lines [14]. The functional palmitoylated sites may provide a new direction for the development of novel therapeutic methods for PAH. Moreover, the palmitoylation levels of specific proteins regulated by cGMP may represent new biomarkers for analyzing prognosis.

\section{Materials And Methods}

Cell culture.

HPASMCs (ScienCell Research Laboratories, California, USA) were isolated from human pulmonary arteries [15] and cultured in the smooth muscle cell (SMC) medium (ScienCell Research Laboratories, Carlsbad, USA) supplemented with $2 \%$ fetal bovine serum and $1 \%$ SMC growth supplement. This study was approved by the Beijing Hospital Ethics Committee (BHEC) with the registration No. of 2017BJYYEC108-05. Hela cells were cultured in Dulbecco's modified Eagle's medium (DMEM) supplemented with 10\% fetal bovine serum and $4.5 \mathrm{mg} / \mathrm{ml}$ glucose. All the cells were incubated at $37^{\circ} \mathrm{C}$ in a humidified normoxia condition $\left(21 \% \mathrm{O}_{2}, 5 \% \mathrm{CO}_{2}\right.$ and $\left.74 \% \mathrm{~N}_{2}\right)$.

Construction of plasmids and DNA transfection. 
To construct the expression plasmids for LETM-Flag, a DNA fragment containing LETM1 was inserted into PCDNA3.1-Flag-C. LETM1 ${ }^{\text {C552A }}$ mutant was constructed by PCR using the primers (Letm1C552A-F: 5'CCTCAGCGATGCCGCCTCTAAGCTG-3' and Letm1C552A-R: 5'-GCGGCATCGCTGAGGATGTCGATTTC-3')

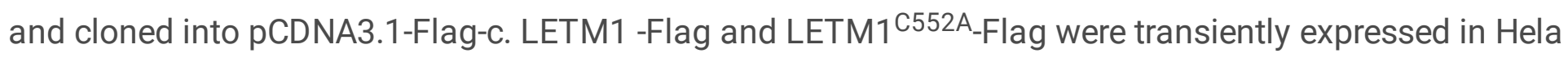
cells and DNA transfection was performed using lipo3000 (Invitrogen) according to the manufacturer's instructions.

Antibodies and reagents.

The antibodies used in the present study were as follows:

rabbit anti-LETM1 (dilution 1:2,000; cat. no. 16024-1-AP), rabbit anti-THEM4 (dilution 1:2,000; cat. no. 14692-1-AP), mouse anti-GAPDH (dilution 1:2,000; cat. no. 60004-1-lg), rabbit anti-Flag (dilution 1:2,000; cat. no. 20543-1-AP), mouse anti-AKT (dilution 1:2,000; cat. no. 60203-1-lg), mouse anti-pS473-AKT (dilution 1:2000; cat. no. 66444-1-lg). All of the antibodies were purchased from Protein Tech Group, Inc.

The reagents used in the present study were as follows:

protease inhibitor cocktail (Roche Group), protein (A/G) ultraLink resin (Thermo Fisher Scientific, Inc.), azide agarose beads (Nanocs), thiopropyl sepharose 6B (Sigma-Aldrich; Merck KGaA), hydroxylamine (Sigma-Aldrich; Merck KGaA), N-ethylmaleimide (NEM; Sigma-Aldrich; Merck KGaA), Tris (2-carboxyethyl)phosphine hydrochloride (TCEP; Sigma-Aldrich; Merck KGaA), Tris [(1-benzyl -1H-1,2,3-triazol-4-yl)methyl]amine (TBTA; Sigma-Aldrich; Merck KGaA), 8-Br-cGMP (Sigma-Aldrich; Merck $\mathrm{KGaA})$.

Immunoprecipitation (IP) and western blot analysis.

HPASMCs were harvested and lysed (50 mM Tris, pH 7.4, $150 \mathrm{mM} \mathrm{NaCl}, 1 \%$ Triton X-100, 10\% glycerol, and protease inhibitor cocktail) on ice for $30 \mathrm{~min}$. IP and western blots were performed as previously described [16].

Protein Spalmitoylation assay in cells.

The HPASMCs were treated with 8-Br-cGMP $(1 \mathrm{mM})$ or $\mathrm{H}_{2} \mathrm{O}$ (control). Hela cells were transfected with

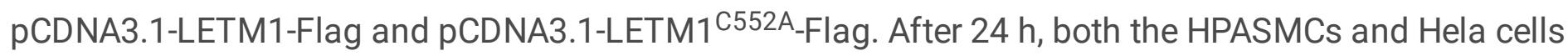
were harvested for S-palmitoylation assay. The S-palmitoylation assay was performed as previously described [17] with minor modifications. In brief, the cells were washed in PBS and lysed in lysis buffer [10 mM sodium phosphate, $2 \mathrm{mM} \mathrm{Na}_{2}$-EDTA, $0.32 \mathrm{M}$ sucrose, $1 \%$ Triton $\mathrm{X}-100$, protease inhibitor cocktail; $\mathrm{pH} 7.4$ ] on ice for $30 \mathrm{~min}$. Then, the lysates were added with N-ethylmaleimide (50 mM) and immunoprecipitated at $4^{\circ} \mathrm{C}$ overnight utilizing protein $\mathrm{A} / \mathrm{G}$ resin preloaded with LETM1 or Flag antibody. Next day, the protein $A / G$ resin was washed three times in lysis buffer and incubated with elution buffer ( $1 \% \mathrm{SDS}, 10 \mathrm{mM}$ sodium phosphate, $2 \mathrm{mM} \mathrm{Na}_{2}$-EDTA, $0.32 \mathrm{M}$ sucrose) at $50^{\circ} \mathrm{C}$ for $5 \mathrm{~min}$ to release 
LETM1, LETM1-Flag or LETM1 ${ }^{\text {C552A }}$-Flag. Eluted samples were divided into two equal portions. One was treated with $1 \mathrm{M}$ hydroxylamine ( $\mathrm{pH} 7.4)$ and thiopropyl sepharose $6 \mathrm{~B}$ and the other was treated with $1 \mathrm{M}$ Tris. $\mathrm{HCl}(\mathrm{pH} 7.4)$ and thiopropyl sepharose 6B as the negative control. After a 2-h incubation at room temperature, sepharose beads were washed three times with washing buffer ( $10 \mathrm{mM}$ sodium phosphate, $2 \mathrm{mM} \mathrm{Na}_{2}$-EDTA, $0.32 \mathrm{M}$ sucrose, $1 \%$ Triton X-100, $500 \mathrm{mM} \mathrm{NaCl}$ and $0.2 \%$ SDS). Western blots was performed to determine the presence of LETM1, LETM1-Flag or LETM1 ${ }^{\mathrm{C} 552 \mathrm{~A}-\text { Flag. }}$

Synthesis of palmitate probe, metabolic labeling and click chemistry reaction.

The $\omega$-alkynyl-palmitate acid analogue, Alk-C16, was synthesized as previously described [18] and dissolved in DMSO to generate a 50-mM stock solution and stored at $80^{\circ} \mathrm{C}$. Before cell treatment, AlkC16 was dissolved in SMC medium supplemented with $5 \%$ BSA (fattyacid free) and $1 \%$ SMC growth supplement at a final concentration of $100 \mu \mathrm{M}$, and then sonicated for $15 \mathrm{~min}$ at room temperature. In addition, an equal volume of DMSO was added to the same medium as a negative control. Then, the seeded HPASMCs were incubated in the above medium containing Alk-C16 or DMSO respectively for $24 \mathrm{~h}$ at $37^{\circ} \mathrm{C}$ with $5 \% \mathrm{CO}_{2}$. Then, the cells were harvested and click chemistry reaction were performed as previously described [18]. In brief, the cells were lysed in $500 \mu \mathrm{l}$ lysis buffer (1\% Nonidet P-40, $150 \mathrm{mM}$ $\mathrm{NaCl}, 100 \mathrm{mM}$ sodium phosphate and protease inhibitor cocktail; $\mathrm{pH} 7.5)$ for $30 \mathrm{~min}$ at $4^{\circ} \mathrm{C}$. Then, the protein extracts were subjected to the click-chemistry reaction for $1 \mathrm{~h}$ at room temperature by the addition of the following reagents in order: $1 \mathrm{mM}$ azide agarose beads, $1 \mathrm{mM}$ TCEP dissolved in water, $0.2 \mathrm{mM}$ TBTA dissolved in DMSO/tert-butanol $(20 / 80 \% \mathrm{v} / \mathrm{v})$ and $1 \mathrm{mM} \mathrm{CuSO}_{4}$ in PBS. Following the reaction, the Alk-C16-conjugated proteins were bound to the azide agarose beads. The beads were washed three times with lysis buffer at room temperature. Then, the proteins bound to the beads were digested for MS analysis.

Proteome analysis.

NanoLCMS/MS analysis, database searching, data processing and statistical analysis were performed as previously described $[17,19]$. The steps were as follows.

An Easy nLC1000 (Thermo Fisher Scientific, Inc.) system combined with the LTQ-Orbitrap-Elite (Thermo Fisher Scientific, Inc.) mass spectrometer were applied for proteomic profiling.

The recorded MS spectra was analyzed by utilizing MaxQuant Software (version 1.5.5.1; https://www.Maxquant.org/). The MS/MS peak list analysis was performed by searching against a forward and reverse version of the UniProtKB/Swiss-Prot human database (generated from version 2017_05; human taxonomy; 20,316 entries; http://www.uniprot.org/). The cutoff of the false discovery rate for peptide and protein identification was set to 0.01 , and only peptides with $\geq 7$ amino acidic residues were analyzed. Label-free quantitation (LFQ) was performed using the MaxQuant software on the identified razor and unique peptides in order to quantify the identified proteins. 
Protein abundances normalized by the LFQ algorithm integrated in MaxQuant were log2-transformed for further analysis. The filtering steps were performed using Microsoft Excel 2010. DanteR (version 1.0.1.1) and Perseus (version 1.5.5.3) were used to perform various types of statistical analysis including log2 transformation, correlation plot, statistical tests and $p$-value adjustments.

Functional analysis.

The functional enrichment analysis of the identified palmitoylated proteins were performed by Metascape web-based platform [20]. Enriched terms with a $p$-value $<0.01$, a minimum count of 3 , and an enrichment factor $>1.5$ were collected and grouped into clusters based on their membership similarities. "Log10 $(P) "$ is the $p$-value in log base 10. Protein-protein interaction (PPI) network construction was performed by STRING database (Search Tool for the Retrieval of Interacting Genes/Proteins, http://string-db.org/) [21].

\section{Results}

Identification transport proteins of palmitoylome using AlkC16 in HPASMCs.

In order to identify the palmitoylated protein profiles of HPASMCs, the cells were metabolic labelled by Alk-C16, an alkynyl-palmitic acid. The proteins modified by the alkynyl-palmitic acid were chemoselectively ligated to azide agarose beads by a click chemistry reaction of $\mathrm{Cu}^{1}$-catalyzed [3+2] azide-alkyne cycloaddition (CuAAC). The application of CuAAC could enrich the palmitoylated proteins on the azide agarose beads. Then, the proteins on the beads were digested by trypsin and subjected to LCMS/MS and palmitoylome analysis. (Fig. 1)

The results of palmitoylome analysis indicated that a total of 1997 proteins were identified, which exhibited a Mascot score $>2(P<0.05)$ and peptide $>2$. The proteins were subjected to LFQ using MaxQuant software and the LFQ values were positively correlated with the palmitoylation levels. Among these 1997 proteins, 1803 proteins were identified to be palmitoylated preliminarily, and their LFQ values were $>0$ in at least 2 replicates. Functional enrichment analysis of 1803 palmitoylated proteins by Metascape showed that 267 of them engaged in transport including vesicle-mediated transport, nucleocytoplasmic transport and transmembrane transport (Fig. 2). Vesicle-mediated transport (R-HSA5653656) included endocytosis, vacuolar transport, phagocytosis, endosomal transport, cytoskeletondependent transport (GO: 0030705, GO: 0008088, GO: 0010970, GO: 0072384, GO: 0099111, GO: 0047496, GO: 0008089, GO: 0048489) and Golgi-ER related transport (GO: 0048193, GO: 0006888, GO: 0006890, R-HSA-199977, R-HSA-8856688, R-HSA-6807878). Nucleocytoplasmic transport (G0: 0006913) engaged in the export from nucleus of RNA, proteins or nitrogen compound. Small molecules transport (R-HSA-382551) involved transmembrane transport of ions, proteins or metabolic products, which we will focus on in the following sections.

Protein-protein interaction network analysis of the small molecules transport proteins. 
There were 52 proteins involved in the small molecules transports (R-HSA-382551) (TableS1). The PPI network of them consisted of 52 nodes and 113 interactions (Fig. 3). PPI combined with GO enrichment analysis showed that the function of the transport proteins concentrated on four portions: iron ion transport (GO: 0006826) (purple marks), mitochondrial transport (GO: 0006839) (green marks), anion transport (GO: 0006820) (red marks), low-density lipoprotein particle clearance (GO: 0034383) (yellow marks) (Fig. 3 and TableS2). Previous studies have shown that mitochondrial function inhibition is the key to the development of PAH [22]. Furthermore, it has never been studied how palmitoylation regulates the activity of mitochondrial transport proteins. The proteins involved in mitochondrial transport (GO: 0006839) included AFG3L2, LETM1 (Leucine zipper-EF-hand-containing transmembrane protein 1), PMPCA, MICU2 (Mitochondrial calcium uniporter protein 2), STOML2 (Stomatin-like protein 2), SLC25A5, and SLC25A6. Both AFG3L2 and PMPCA contribute to the mitochondrial proteins import through the cleavage of their peptides $[23,24]$. LETM1 is an antiporter that mediated proton-dependent calcium efflux from mitochondrion [25]. MICU2 regulates calcium uptake [26]. SLC25A5 and SLC25A6 mediate the output of ATP and provide fuel for cells [27]. Next, we further verified the palmitoylation of the mitochondrial transport proteins by S-palmitoylation assay in vitro and screened the candidate proteins, whose palmitoylation level was regulated by cGMP in HPASMCs.

LETM1 is a novel palmitoylated protein at C-terminus.

To confirm the palmitoylated proteins involved in mitochondrial transport, we performed S-palmitoylation assay in HPASMCs. As shown in Fig. 4.A, LETM1 was detected to be associated with the thiopropyl beads in the presence of hydroxylamine compared to the negative control of Tris. $\mathrm{HCl}$. The result indicated that palmitoylation did exist in LETM1 and the level of palmitoylation was very high, which was consistent with the results of MS. In addition, we also detected the palmitoylation of LETM1 in Hela cells by the same assay and obtained the same result as that in HPASMCs (Fig. 4.B).

Next, we identified the specific palmitoylated cysteine residues of LETM1. As shown in Fig. 4.C and 5.E, the mitochondrial target sequence (1aa-114aa) was located at the N-terminal, TM1 (206aa-228aa) and TM2 (413aa-421aa) were the transmembrane domains of LETM1, and the N-terminal and C-terminal were located in the mitochondrial matrix. The domain analysis of LETM1 was original from Gayathri et al [28].

There was only cysteine 552 at the C-terminus of LETM1, which was conserved among human, mouse, rat and zebrafish (Fig. 4.D). To confirm whether the cysteine 552 was required for LETM1 palmitoylation, we generated a mutant form of LETM1 in which the cysteine (C) site was mutated to alanine (A). Then, we over-expressed LETM1-Flag and LETM1C552A-Flag in Hela cells and performed S-palmitoylation assay respectively. As shown in Figure 4E, the palmitylation level of LETM1C552A was lower than that of wild type LETM1.

Therefore, we conclude that LETM1 is palmitoylated at cysteine 552 , which is located in the cytoplasmic domain at C-terminus.

cGMP inhibits the palmitoylation of LETM1 and the activity of AKT. 
Since LETM1 was palmitoylated, we tested whether cGMP regulated the palmitoylation level of LETM1. As shown in Figure 5.A, 8-Br-cGMP inhibited the palmitoylation level of LETM1 dramatically compared to the negative control, indicating that CGMP was a negative regulator of the palmitoylation of LETM1. Based on the result, we wondered how cGMP regulated the downstream biological processes by inhibiting the palmitoylation of LETM1. The PPI network analysis of LETM1 showed that LETM1 engaged in mitochondrial transport, which was involved in two aspects (Fig. 5.B). One was to regulate mitochondrial calcium ion transport (red marks) by cooperating with MICU, MICU1, MICU2, SLC8B1, etc. The other was to regulate AKT activity via the cooperation with THEM4 (acyl-coenzyme A thioesterase) (Fig. 5.B). According to the analysis, we carried out co-immunoprecipitation experiments in HPASMCs and found that endogenous LETM1 and THEM4 could be immunoprecipitated in HPASMCs (Fig. 5.C). Considering that THEM4 was involved in regulating the activity of AKT [29], we tested whether cGMP affected the activity of AKT in HPASMCs. After 8-Br-cGMP treatment, the phosphorylation level of AKT was significantly downregulated (Fig. 5.D). The result indicates that cGMP is the negative regulator of AKT activity. Taken together, we conclude that CGMP inversely regulates the palmitoylation of LETM1 and downregulates the activity of AKT.

\section{Discussion}

Palmitoylation at cytoplasmic domains is essential for transmembrane transport proteins, which is involved in membrane anchoring for regulating protein-protein interactions, the function of the proximate transmembrane domains, and so on [11]. Among the palmitoylated transmembrane transport proteins which have been studied so far, the majority of them localize to the plasma membrane, such as ion channels [12,13], while almost rarely in the mitochondrial membrane. Previous studies found that LETM1, a protein in the inner mitochondrial membrane, participates in a variety of cellular functions, such as mitochondrial metabolism and cellular signaling [28].

THEM4 inhibits the phosphorylation of AKT by binding its hydrophobic motif [29] and interacts with LETM1 to regulate glucose metabolism in liver cells from obese and high fat diet-fed mice [30]. However, there is no evidence to explain that how LETM1 regulates the AKT activity through THEM4, except that the LETM1 expression is inversely correlated with the THEM4 expression and positively correlated with the AKT activity [30]. In this study, we found that LETM1 was palmitoylated by the palmitoylome with click-chemistry-probes (Fig. 1). More importantly, cGMP could inhibit the palmitoylation level of LETM1 in HPASMCs (Fig. 5.A). Our study also found that endogenous LETM1 could be immunoprecipitated with THEM4 in HPASMCs (Fig. 5.C). Meanwhile, we found that cGMP could inhibit the palmitoylation level of LETM1 and downregulate the AKT activity in HPASMCs (Fig. 5.A and 5.D). Based on the results, we speculated that LETM1 prevented THME4 exporting from mitochondria to cytoplasm through palmitoylation, while cGMP promoted the release of THEM4 to inhibit the AKT activity by inhibiting the palmitoylation of LETM1 (Fig. 5.E). However, in view of the fact that the primary HPASMCs were difficult to obtain and varied greatly among individuals, we need to conduct in vivo experiments in mouse models to verify the speculation. In addition, we also obtained other palmitoylated proteins involved in mitochondrial transport and would pay attention to them in the future. 


\section{Conclusion}

In this work, we found that LETM1 was a palmitoylated protein in the inner mitochondrial membrane. Moreover, cGMP could inhibit the palmitoylation level of LETM1 and suppress the AKT activity in HPASMCs. Our studies not only explored the nature of the interaction between LETM1 and THEM4 but also unraveled the new advance in understanding of cGMP signaling pathway in PAH. The palmitoylated site of LETM1 may provide new directions for the development of novel therapies for PAH. Moreover, the palmitoylated level of LEMT1 may represent a new biomarker for analyzing prognosis.

\section{Declarations}

\section{Statement of Ethics}

This study was approved by the Beijing Hospital Ethics Committee (BHEC) with the registration No. of 2017BJYYEC-108-05.

\section{Conflict of Interest Statement}

None.

\section{Authors' contributions}

Wenqing Li: conceptualization, data curation, formal analysis, methodology, software and writingoriginal draft. Wei Huang: data curation, methodology. Ran Miao: methodology, investigation. Lihui Zou: funding acquisition, supervision. Fei Xiao: software, funding acquisition, writing-review \& editing. All authors read and approved the manuscript and agree to be accountable for all aspects of the research in ensuring that the accuracy or integrity of any part of the work are appropriately investigated and resolved.

\section{Acknowledgements}

The proteomic experiments were performed using the mass spectrometry facility of The State Key Laboratory of Membrane Biology, Institute of Zoology, Chinese Academy of Sciences.

\section{Funding}

This work was supported by the National Key Research and Development Program of China (Grant 2018YFC2000505) (sponsor: Fei Xiao), National Natural Science Foundation of China (Grants 81871107) (sponsor: Lihui Zou) and National Natural Science Foundation of China (Grants 81870048) (sponsor: Fei Xiao).

\section{Data Availability Statement}

All the data are available and can be found. 


\section{References}

1. Beavo JA, Brunton LL (2002) Cyclic nucleotide research -- still expanding after half a century. Nat Rev Mol Cell Biol 3:710-718. doi: 10.1038/nrm911

2. Chen CN, Watson G, Zhao L (2013) Cyclic guanosine monophosphate signalling pathway in pulmonary arterial hypertension. Vascul Pharmacol 58:211-218. doi: 10.1016/j.vph.2012.09.001

3. Murad F (2006) Shattuck Lecture. Nitric oxide and cyclic GMP in cell signaling and drug development. N Engl J Med 355:2003-2011. doi: 10.1056/NEJMsa063904

4. Schermuly RT, Ghofrani HA, Wilkins MR, Grimminger F (2011) Mechanisms of disease: pulmonary arterial hypertension. Nat Rev Cardiol 8:443-455. doi: 10.1038/nrcardio.2011.87

5. Jiang H, Zhang X, Chen X, Aramsangtienchai P, Tong Z, Lin H (2018) Protein Lipidation: Occurrence, Mechanisms, Biological Functions, and Enabling Technologies. Chem Rev 118:919-988. doi: 10.1021/acs.chemrev.6b00750

6. Chen B, Sun Y, Niu J, Jarugumilli GK, Wu X (2018) Protein Lipidation in Cell Signaling and Diseases: Function, Regulation, and Therapeutic Opportunities. Cell Chem Biol 25:817-831. doi: 10.1016/j.chembiol.2018.05.003

7. Thinon E, Hang HC (2015) Chemical reporters for exploring protein acylation. Biochem Soc Trans 43:253-261. doi: 10.1042/bst20150004

8. Chen JJ, Fan Y, Boehning D (2021) Regulation of Dynamic Protein S-Acylation. Front Mol Biosci 8:656440. doi: 10.3389/fmolb.2021.656440

9. Ko PJ, Dixon SJ (2018) Protein palmitoylation and cancer. EMBO Rep 19. doi: 10.15252/embr.201846666

10. Lin DTS, Davis NG, Conibear E (2017) Targeting the Ras palmitoylation/depalmitoylation cycle in cancer. Biochem Soc Trans 45:913-921. doi: 10.1042/bst20160303

11. Charollais J, Van Der Goot FG (2009) Palmitoylation of membrane proteins (Review). Mol Membr Biol 26:55-66. doi: 10.1080/09687680802620369

12. McCarthy AE, Yoshioka C, Mansoor SE (2019) Full-Length P2X(7) Structures Reveal How Palmitoylation Prevents Channel Desensitization. Cell 179:659-670e13. doi:

10.1016/j.cell.2019.09.017

13. Gök C, Fuller W (2020) Regulation of NCX1 by palmitoylation. Cell Calcium 86:102158. doi: 10.1016/j.ceca.2019.102158

14. Hannoush RN, Sun J (2010) The chemical toolbox for monitoring protein fatty acylation and prenylation. Nat Chem Biol 6:498-506. doi: 10.1038/nchembio.388

15. Wang G, Liu X, Meng L, Liu S, Wang L, Li J, Cui C, Meng J, Hu S, Wei Y (2014) Up-regulated lipocalin-2 in pulmonary hypertension involving in pulmonary artery SMC resistance to apoptosis. Int J Biol Sci 10:798-806. doi: 10.7150/ijbs.9470

16. Chen D, Wang Q, Huang H, Xia L, Jiang X, Kan L, Sun Q, Chen D (2009) Effete-mediated degradation of Cyclin A is essential for the maintenance of germline stem cells in Drosophila. Development 
136:4133-4142. doi: $10.1242 /$ dev.039032

17. Li W, Zhang J, Zou L, Cui J, Su F, Jin J, Xiao F, Liu M, Zhao G (2019) Palmitoylome profiling indicates that androgens regulate the palmitoylation of a-tubulin in prostate cancer-derived LNCaP cells and supernatants. Oncol Rep 42:2788-2796. doi: 10.3892/or.2019.7333

18. Hannoush RN, Arenas-Ramirez N (2009) Imaging the lipidome: omega-alkynyl fatty acids for detection and cellular visualization of lipid-modified proteins. ACS Chem Biol 4:581-587. doi: $10.1021 / \mathrm{cb} 900085 z$

19. Liu NQ, Braakman RB, Stingl C, Luider TM, Martens JW, Foekens JA, Umar A (2012) Proteomics pipeline for biomarker discovery of laser capture microdissected breast cancer tissue. J Mammary Gland Biol Neoplasia 17:155-164. doi: 10.1007/s10911-012-9252-6

20. Zhou Y, Zhou B, Pache L, Chang M, Khodabakhshi AH, Tanaseichuk O, Benner C, Chanda SK (2019) Metascape provides a biologist-oriented resource for the analysis of systems-level datasets. Nat Commun 10:1523. doi: 10.1038/s41467-019-09234-6

21. Szklarczyk D, Franceschini A, Wyder S, Forslund K, Heller D, Huerta-Cepas J, Simonovic M, Roth A, Santos A, Tsafou KP, Kuhn M, Bork P, Jensen LJ, von Mering C (2015) STRING v10: protein-protein interaction networks, integrated over the tree of life. Nucleic Acids Res 43:D447-D452. doi: 10.1093/nar/gku1003

22. Bhansali S, Sohi K, Dhawan V (2021) Hypoxia-induced mitochondrial reactive oxygen species (mtROS) differentially regulates smooth muscle cell (SMC) proliferation of pulmonary and systemic vasculature. Mitochondrion 57:97-107. doi: 10.1016/j.mito.2020.11.012

23. Baderna V, Schultz J, Kearns LS, Fahey M, Thompson BA, Ruddle JB, Huq A, Maltecca F (2020) A novel AFG3L2 mutation close to AAA domain leads to aberrant OMA1 and OPA1 processing in a family with optic atrophy. Acta Neuropathol Commun 8:93. doi: 10.1186/s40478-020-00975-w

24. Jobling RK, Assoum M, Gakh O, Blaser S, Raiman JA, Mignot C, Roze E, Dürr A, Brice A, Lévy N, Prasad C, Paton T, Paterson AD, Roslin NM, Marshall CR, Desvignes JP, Roëckel-Trevisiol N, Scherer SW, Rouleau GA, Mégarbané A, Isaya G, Delague V, Yoon G (2015) PMPCA mutations cause abnormal mitochondrial protein processing in patients with non-progressive cerebellar ataxia. Brain 138:1505-1517. doi: 10.1093/brain/awv057

25. Jiang D, Zhao L, Clapham DE (2009) Genome-wide RNAi screen identifies Letm1 as a mitochondrial $\mathrm{Ca} 2+/ \mathrm{H}+$ antiporter. Science 326:144-147. doi: 10.1126/science.1175145

26. Patron M, Checchetto V, Raffaello A, Teardo E, Vecellio Reane D, Mantoan M, Granatiero V, Szabò I, De Stefani D, Rizzuto R (2014) MICU1 and MICU2 finely tune the mitochondrial Ca2+ uniporter by exerting opposite effects on MCU activity. Mol Cell 53:726-737. doi: 10.1016/j.molcel.2014.01.013

27. Bertholet AM, Chouchani ET, Kazak L, Angelin A, Fedorenko A, Long JZ, Vidoni S, Garrity R, Cho J, Terada N, Wallace DC, Spiegelman BM, Kirichok $\mathrm{Y}(2019) \mathrm{H}(+)$ transport is an integral function of the mitochondrial ADP/ATP carrier. Nature 571:515-520. doi: 10.1038/s41586-019-1400-3

28. Natarajan GK, Mishra J, Camara AKS, Kwok WM (2021) LETM1: A Single Entity With Diverse Impact on Mitochondrial Metabolism and Cellular Signaling. Front Physiol 12:637852. doi: 
10.3389/fphys.2021.637852

29. Maira SM, Galetic I, Brazil DP, Kaech S, Ingley E, Thelen M, Hemmings BA (2001) Carboxyl-terminal modulator protein (CTMP), a negative regulator of PKB/Akt and v-Akt at the plasma membrane. Science 294:374-380. doi: 10.1126/science.1062030

30. Park J, Li Y, Kim SH, Yang KJ, Kong G, Shrestha R, Tran Q, Park KA, Jeon J, Hur GM, Lee CH, Kim DH, Park J (2014) New players in high fat diet-induced obesity: LETM1 and CTMP. Metabolism 63:318327. doi: 10.1016/j.metabol.2013.10.012

\section{Figures}



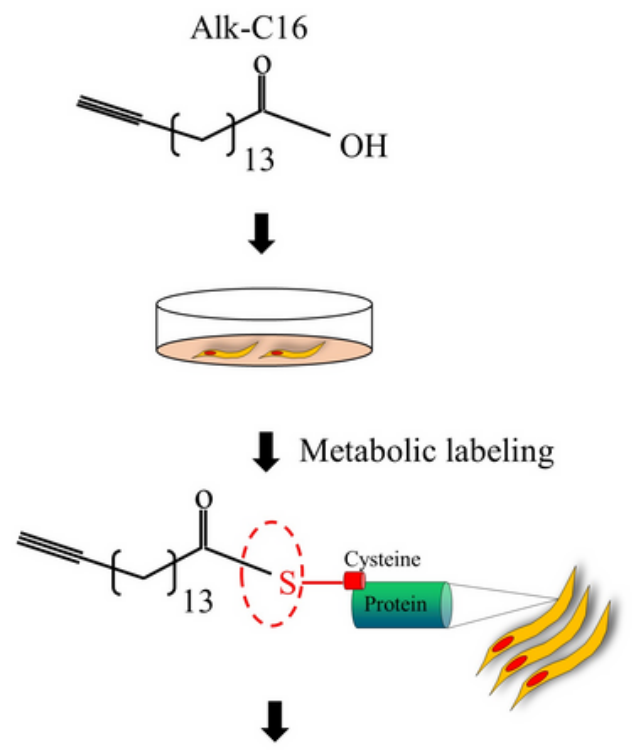

Cell lysis

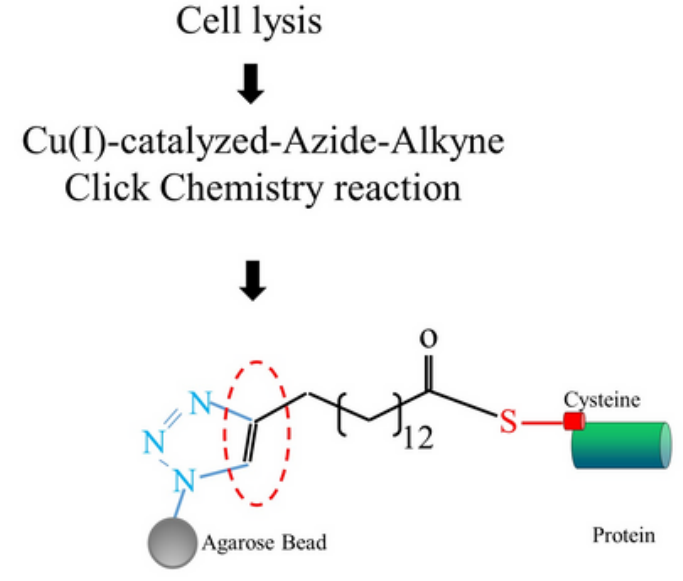

$\downarrow$ Tryptic digest on beads
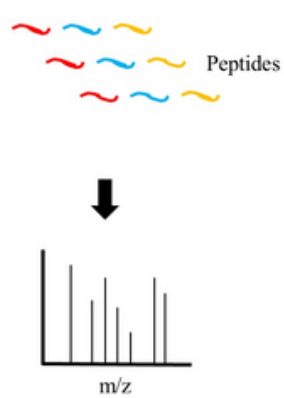

LC-MS/MS

\section{Figure 1}

Experimental strategy for the identification of the palmitoylome using click chemistry reaction in HPASMCs. Cultured cells were incubated with Alk-C16, an alkynyl-palmitic acid. The Alk-C16-conjugated proteins were bound to the azide agarose beads by click chemistry reaction. The proteins were trypsin digested and subjected to LC-MS/MS and palmitoylome analysis. 


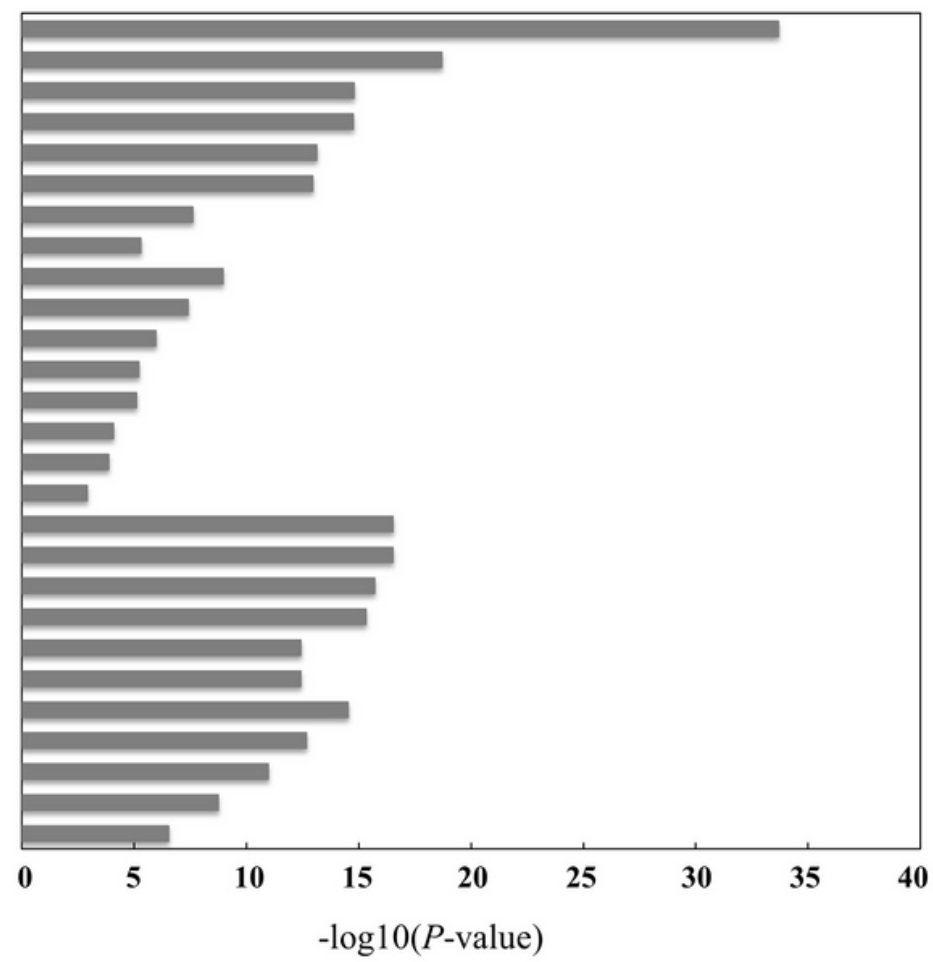

R-HSA-5653656 Vesicle-mediated transport

R-HSA-8856688 Golgi-to-ER retrograde transport

R-HSA-199977 ER to Golgi anterograde transport

R-HSA-6807878 COPI-mediated anterograde transport

R-HSA-948021 Transport to the Golgi and subsequent modification GO:0048193 Golgi vesicle transport

GO:0006888 ER to Golgi vesicle-mediated transport

GO:0006890 retrograde vesicle-mediated transport, Golgi to ER

GO:0030705

GO:0008088

retrograde vesicle-mediated transport, Golgi to
cytoskeleton-dependent intracellular transport

GO:0010970

GO:0072384

GO:0099111

axo-dendritic transport

transport along microtubule

organelle transport along microtubule

GO:0047496

GO:0008089

microtubule-based transport

vesicle transport along microtubule

GO:0048489

anterograde axonal transport

GO:0050657

GO:0050658

GO:0015931

GO:0051028

GO:0006913

GO:0051169

synaptic vesicle transport

nucleic acid transport

RNA transport

nucleobase-containing compound transport

mRNA transport

nucleocytoplasmic transport

R-HSA-382556 ABC-family proteins mediated transport

R-HSA-5619084 ABC transporter disorders

R-HSA-382551 Transport of small molecules

R-HSA-5619115 Disorders of transmembrane transporters GO:1901998 toxin transport

$-\log 10(P$-value $)$

\section{Figure 2}

Enriched terms of the transport-related proteins of palmitoylome in HPASMCs.

Functional enrichment of 1803 palmitoylated proteins were performed by Metascape web-based platform and the transport protein clusters were screened. Enriched terms with a $p$-value $<0.01$, a minimum count of 3 , and an enrichment factor $>1.5$ are collected and grouped into clusters based on their membership similarities. "Log10 $(P)$ " is the $p$-value in log base 10. 


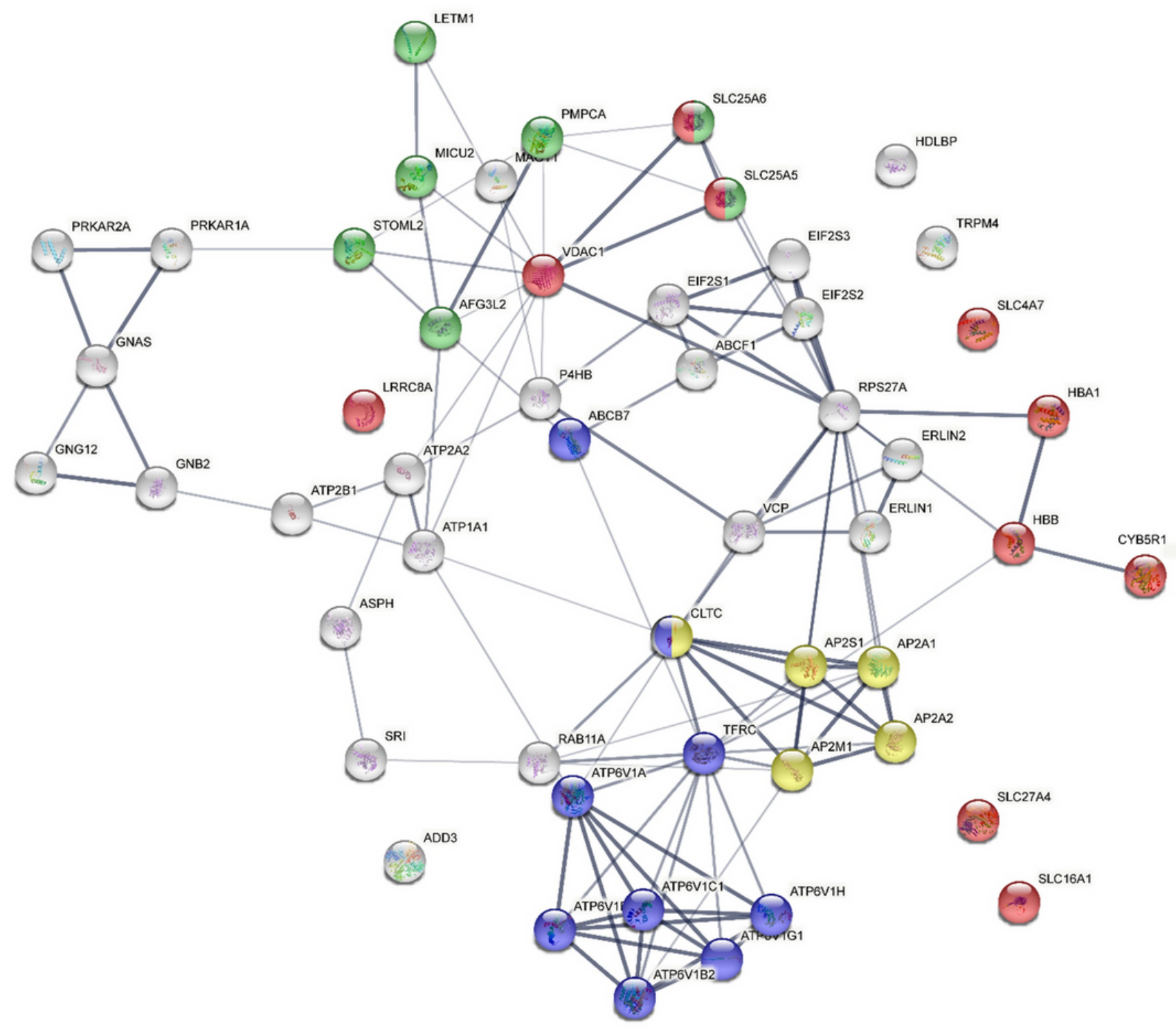

Mitochondrial transport $\bigcirc$ Low-density lipoprotein particle clearance

Iron ion transport Anion transport

Figure 3

Protein-protein interaction network constructed with the small molecules transport proteins. Nodes represent proteins, and the number of nodes is 52 . Edges represent protein-protein associations, and the number of edges is 113. Line thickness indicates the strength of data support. Average local clustering coefficient: 0.524 , PPI enrichment $p$-value: $<1.0 \mathrm{e}-16$. Different colors of the nodes represent different GO terms. 
A

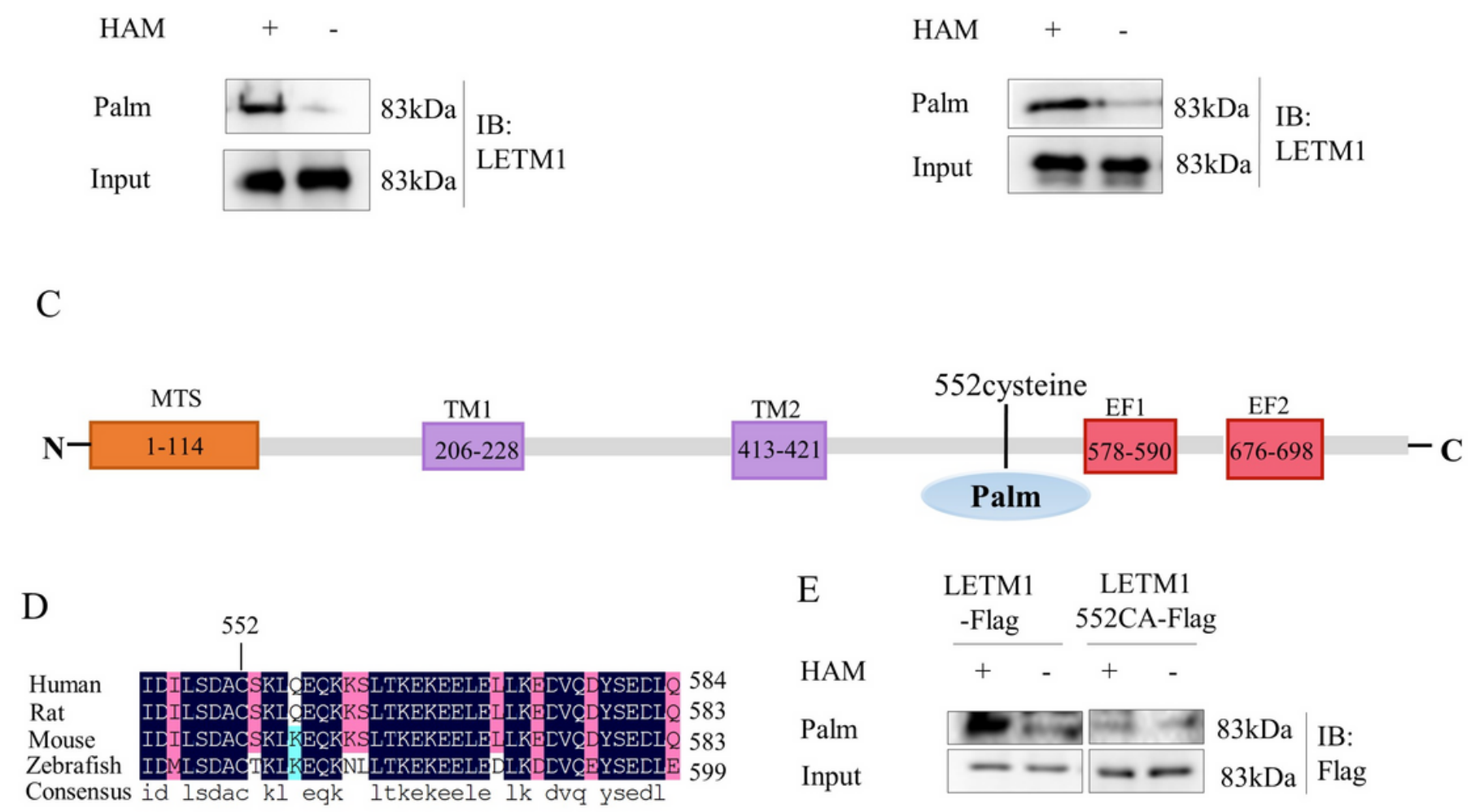

\section{Figure 4}

LETM1 is a novel palmitoylated protein at C-terminus. LETM1 palmitoylation was determined using an Spalmitoylation assay in HPASMCs (A) and in Hela cells (B). (C) Domain schematic of LETM1 including MTS (mitochondrial targeting sequence), TM1 (conserved transmembrane domain 1), TM2 (putative transmembrane domain 2), EF1, EF2 (non-canonical $\mathrm{Ca}^{2+}{ }^{2+b i n d i n g ~ E F-h a n d ~ d o m a i n s) . ~(D) ~ A l i g n m e n t ~ o f ~}$ LETM1 amino acid sequence with its homolog for conserved cysteines at C-terminus. Alignment of the LETM1 sequence in human (Uniprot accession number: 095202) with that of LETM1 in mouse (Uniprot accession number: Q9Z2I0), LETM1 in Rat (Uniprot accession number: Q5XIN6), LETM1 in Zebrafish (Uniprot accession number: Q1LY46). Highly conserved residues are shown in black. (E) LETM1 was palmitoylated at cysteine 552. Hela cells were transiently expressed LETM1-Flag (wild type) or

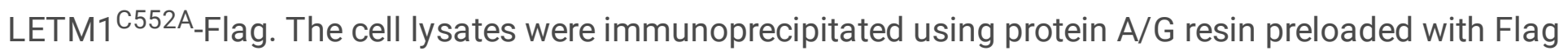
antibody, and then subjected to S-palmitoylation assay to measure palmitoylation level of LETM1 and its mutant. HAM and Palm are abbreviations for hydroxylamine and palmitoylation, respectively. 
A

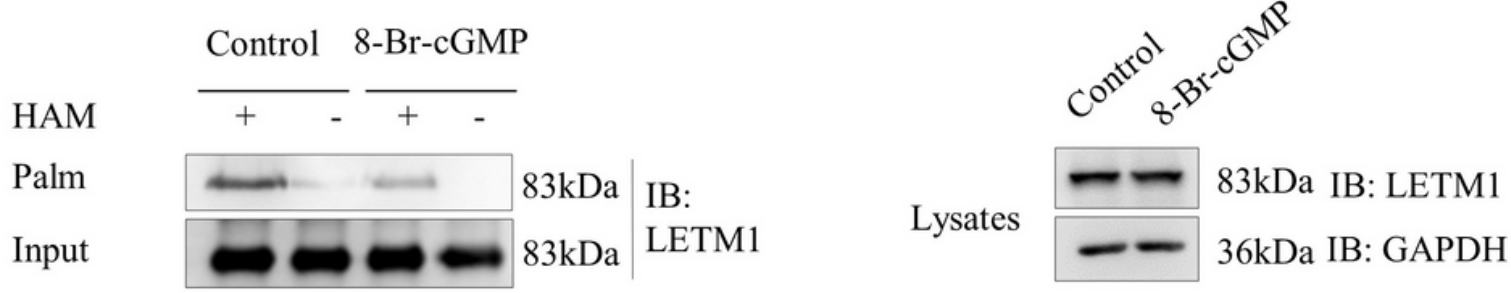

B

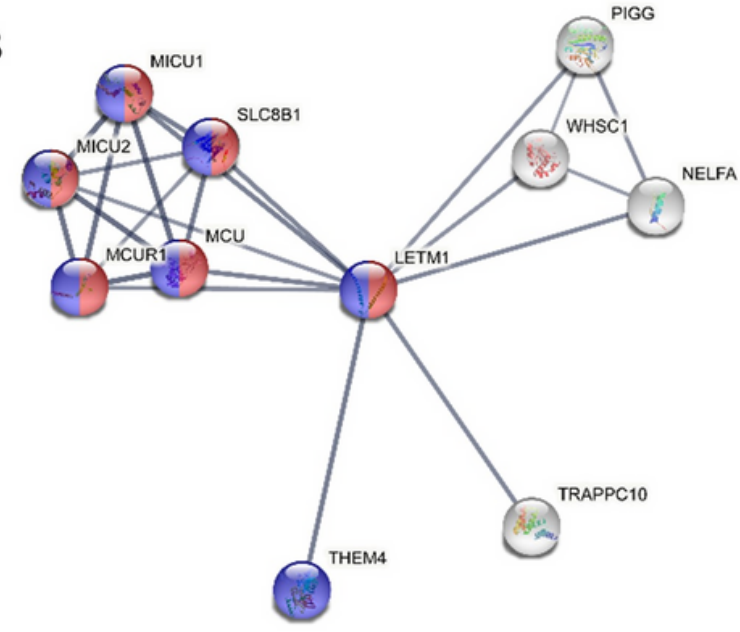

$\mathrm{C}$

IP

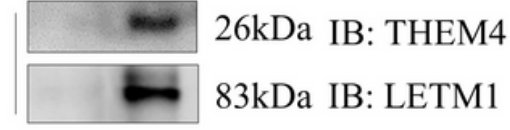

Lysates

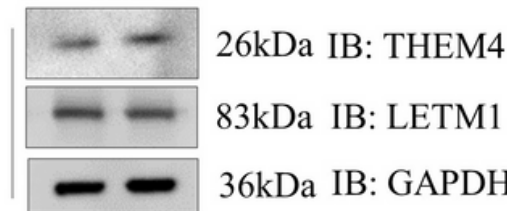

Mitochondrial calcium ion transmembrane transport

Mitochondrial transport

$\mathrm{D}$

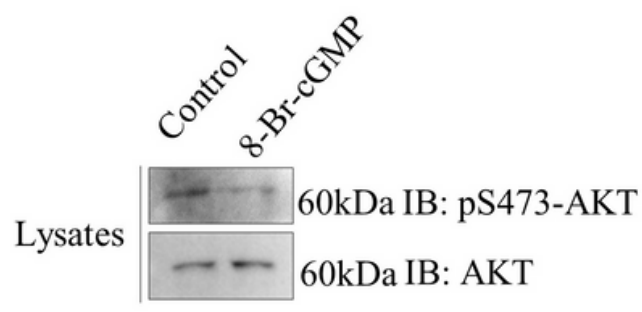

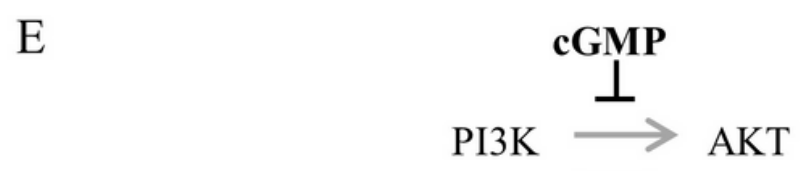

\section{Figure 5}

cGMP inhibits the palmitoylation of LETM1 and suppresses the activity of AKT. (A) 8-Br-cGMP inhibited the palmitoylation of LETM1 in HPASMCs determined by S-palmitoylation assay. HPASMCs were treated with $\mathrm{H}_{2} \mathrm{O}$ (as a control) or 8-Br-cGMP. Cell lysates were immunoprecipitated using protein $\mathrm{A} / \mathrm{G}$ resin preloaded with LETM1 antibody and then subjected to S-palmitoylation assay. HAM and Palm are abbreviations for hydroxylamine and palmitoylation, respectively. (B) The biological process of LETM1 was analyzed by PPI combined with GO enrichment. (C) Endogenous LETM1 and THEM4 were immunoprecipitated in HPASMCs. Cell lysates were immunoprecipitated using protein A/G resin 
preloaded with LETM1 antibody. Western blots were performed to analyze the presence of THEM4. (D) 8Br-cGMP inhibited the activity of AKT. HPASMCs were treated with $\mathrm{H}_{2} \mathrm{O}$ (as a control) or 8-Br- cGMP, and then lysed for analyzing the presence of pS473-AKT and AKT by western blot. (E) Mechanistic summary of the effects of cGMP on LETM1 palmitoylation and AKT activity. The dotted lines indicate that the function of LETM1 is unknown.

\section{Supplementary Files}

This is a list of supplementary files associated with this preprint. Click to download.

- TableS1.docx

- Tables2.docx 